\title{
Prevalence of sarcopenia in community- dwelling older adults using the updated EWGSOP2 definition according to kidney function and albuminuria
}

\section{The Screening for CKD among Older People across Europe (SCOPE) study}

Rafael Moreno-Gonzalez ${ }^{1}$, Xavier Corbella ${ }^{1,2}$, Francesco Mattace-Raso ${ }^{3}$, Lisanne Tap ${ }^{3}$, Cornel Sieber ${ }^{4}$, Ellen Freiberger ${ }^{4}$, Tomasz Kostka ${ }^{5}$, Agnieszka Guligowska ${ }^{5}$, Itshak Melzer ${ }^{6}$, Yehudit Melzer ${ }^{6,7}$, Axel C. Carlsson ${ }^{8,9}$, Johan Ärnlö $v^{8,9,10}$, Regina Roller-Wirnsberger ${ }^{11}$, Gerhard Wirnsberger ${ }^{11}$, Pedro Gil ${ }^{12}$, Sara Lainez Martinez ${ }^{12}$, Paolo Fabbietti ${ }^{13}$, Andrea Corsonello ${ }^{13,14^{*}}$, Fabrizia Lattanzio ${ }^{14}$, Francesc Formiga ${ }^{1}$ and on behalf of SCOPE investigators

\footnotetext{
Abstract

Background: Loss of muscle mass and function may be more pronounced in older adults with chronic kidney disease (CKD) and with albuminuria. Thus, we investigated the prevalence of sarcopenia among community-dwelling older adults according to kidney function and grade of albuminuria. We also explored differences in the prevalence of sarcopenia according to three different equations for the estimation of glomerular filtration rate (eGFR).

Methods: A cross-sectional analysis of 1420 community-dwelling older adults ( $\geq 75$ years old) included in the SCOPE study, a multicenter prospective cohort study, was conducted. Comprehensive geriatric assessment including short physical performance battery (SPPB), handgrip strength test and bioelectrical impedance analysis (BIA) was performed. Sarcopenia was defined using the updated criteria of the European Working Group on Sarcopenia in Older People (EWGSOP2). eGFR was calculated using Berlin Initiative Study (BIS), Chronic Kidney Disease Epidemiological Collaboration (CKD-EPI) and Full Age Spectrum (FAS) equations, and urinary albumin-to-creatinine ratio (ACR) was collected to categorize CKD according to Kidney Disease Improving Global Outcomes guidelines.

(Continued on next page)
}

\footnotetext{
* Correspondence: a.corsonello@inrca.it

${ }^{13}$ Laboratory of Geriatric Pharmacoepidemiology and Biostatistics, IRCCS INRCA, Via S. Margherita 5, 60124 Ancona, Italy

${ }^{14}$ Italian National Research Center on Aging (IRCCS INRCA), Ancona, Fermo and Cosenza, Italy

Full list of author information is available at the end of the article
}

C C The Author(s). 2020 Open Access This article is licensed under a Creative Commons Attribution 4.0 International License, which permits use, sharing, adaptation, distribution and reproduction in any medium or format, as long as you give appropriate credit to the original author(s) and the source, provide a link to the Creative Commons licence, and indicate if changes were made. The images or other third party material in this article are included in the article's Creative Commons licence, unless indicated otherwise in a credit line to the material. If material is not included in the article's Creative Commons licence and your intended use is not permitted by statutory regulation or exceeds the permitted use, you will need to obtain permission directly from the copyright holder. To view a copy of this licence, visit http://creativecommons.org/licenses/by/4.0/ The Creative Commons Public Domain Dedication waiver (http://creativecommons.org/publicdomain/zero/1.0/) applies to the data made available in this article, unless otherwise stated in a credit line to the data. 
(Continued from previous page)

Results: Median age was 79.5 years (77.0-83.0), 804 (56.6\%) were women. Using EWGSOP2 definition, 150 (10.6\%) participants met diagnostic criteria for sarcopenia. Moreover, 85 (6\%) participants had severe sarcopenia. Sarcopenia was more prevalent in participants with more advanced stages of CKD according to BIS eq. (9.6\% in stages 1 and 2 and 13.9\% in stages 3a, 3b and 4, $p=0.042)$, and also according to CKD-EPI (9.8\% vs. 14.2\%, $p=0.042)$ and FAS although not reaching statistical signification ( $9.8 \%$ vs. $12.7 \%, p=0.119)$. Thus, differences in prevalence are observed among CKD categories as estimated by different equations. Prevalence of sarcopenia was also higher with increasing albuminuria categories: $9.3 \%$ in normoalbuminuric, $13.2 \%$ in microalbuminuric and $16.8 \%$ in macroalbuminuric participants, $(p=0.019)$.

Conclusions: Sarcopenia is common among community-dwelling older adults, especially among those with more advanced CKD categories, with prevalence estimates differing slightly depending on the equation used for the estimation of eGFR; as well as among those with higher albuminuria categories.

Keywords: Older adults, Sarcopenia, Chronic kidney disease, Albuminuria, EWGSOP2, Estimated glomerular filtration rate

\section{Background}

The ageing process is characterised by quantitative and qualitative changes in body composition. Those affecting skeletal muscle mass and function are among the most relevant [1]. Sarcopenia is a muscle disease rooted in adverse muscle changes that accrue across a lifetime and is common among older adults, while it can also occur earlier in life. It represents a major cause of falls, is associated with other adverse health outcomes and predicts disability and mortality in older people [2]. Many definitions of sarcopenia have been proposed, even through various consensus from several societies such as the European Society of Clinical Nutrition (ESPEN) [3], European Working Group on Sarcopenia in Older People (EWGSOP) [4], International Working Group on Sarcopenia (IWGS) [5], Society on Sarcopenia, Cachexia and Wasting Disorders (SCWD) [6], Asian Working Group for Sarcopenia (AWGS) [7] and Foundation for the National Institutes of Health (FNIH) [8]. Differences exist in the criteria used for an operational definition, the tools used to measure them including different methods to adjust for body size, and the cut-off points used for each variable; thus leading to heterogeity in research studies and clinical practice, and to differences in prevalence estimates [9-11]. Recently, the EWGSOP updated the original definition of sarcopenia (EWGSOP2) [12], considering muscle strength as a central determinant of sarcopenia, suggesting specific tools and cut-off points for each variable defining sarcopenia, and also proposing a clinical algorithm for the identification, diagnosis and severity assessment of sarcopenia in a stepwise fashion. Thus, sarcopenia is considered probable when low muscle strength is detected, confirmed when low muscle mass is also evidenced, and severe when additionally low physical performance is present.

Chronic kidney disease (CKD) prevalence increases with age, and is often associated with additional comorbid conditions. In patients with CKD, loss of muscle mass is much more intense and the first signs of sarcopenia are observed in younger patients than it is expected [13]. Sarcopenia is more common among patients in the most advanced stages of CKD and is significantly associated with glomerular filtration rate (GFR) decline [14, 15]. Moreover, a bidirectional interaction between sarcopenia and albuminuria has been reported, sarcopenia is more prevalent in individuals with albuminuria than in those without; furthermore, increased albuminuria is independently associated with low muscle mass in patients with type 2 diabetes $[16,17]$.

The present study aimed to investigate the prevalence of sarcopenia as derived from the recently updated EWGSOP2 definition among a clinically relevant source of community-dwelling older adults, and to further assess its distribution according to different kidney function categories and grades of albuminuria, in the frame of the Screening for Chronic Kidney Disease among Older People across Europe (SCOPE) study. A second objective was to explore possible differences between three distinct equations for the estimation of GFR and the prevalence of sarcopenia.

\section{Methods \\ Study design and participants}

This cross-sectional study used data from the SCOPE study (European Grant Agreement no. 436849), a multicenter 2-year prospective cohort study involving patients older than 75 years attending geriatric and nephrology outpatient services in participating institutions in Austria, Germany, Israel, Italy, the Netherlands, Poland and Spain. Methods of the SCOPE study have been extensively described elsewhere [18]. Patients were requested to sign a written informed consent before entering the study. The study protocol was approved by ethics committees at all participating institutions, and complies with the Declaration of Helsinki and Good Clinical Practice Guidelines. Briefly, exclusion criteria were: a. Age $<75$ years; b. End-stage renal disease (ESRD) $\left(\mathrm{eGFR}<15 \mathrm{ml} / \mathrm{min} / 1.73 \mathrm{~m}^{2}\right)$ or dialysis at the 
time of enrollment; c. History of solid organ or bone marrow transplantation; d. Active malignancy within 24 months prior to screening or metastatic cancer; e. Life expectancy less than 6 months; f. Severe cognitive impairment (Mini Mental State Examination < 10); g. Any medical or other reason (e.g. known or suspected inability of the patient to comply with the protocol procedure) in the judgement of the investigators, that the patient is unsuitable for the study; h. Unwilling to provide consent and those who cannot be followed-up. After obtaining written informed consent, all participants underwent an extensive baseline visit including routine laboratory analysis and comprehensive geriatric assessment (CGA). The baseline visit was followed by follow-up visits at 12 and 24 months with intermediate phone contacts at 6 and 18 months. Only baseline data were used in the present study.

Overall, 2461 participants were intially enrolled in the study. Of them, 204 participants with missing serum creatinine and/or urinary albumin-to-creatinine ratio (ACR) were excluded, thus leaving a sample of 2257 participants to be included in the initial analysis. For the aim of the present study, only those participants in whom sarcopenia could be assessed in its three components (i.e., muscle strength, muscle mass and physical performance) were considered. Data for muscle strength, as assessed by grip strength; muscle mass, as assessed by bioelectrical impedance analysis (BIA); and physical performance, as assessed by the Short Physical Performance Battery (SPPB) were available for 2138 (94.7\%), 1462 (64.8\%) and 2256 (99.9\%) participants respectively. Participants with missing data mainly included those physically unable or unsteady, those presenting arthralgia or arthritis, those with an implanted cardioverterdefibifrillator or pacemaker, or those not assessed due to any other safety reason in the judgement of the investigators. 1420 participants were finally included, for whom demographic and clinical characteristics were analysed. Anthropometric measures were collected and body mass index (BMI) was calculated as recommended in the ESPEN guidelines [19]. Cognitive function was assessed with the Mini Mental State Examination (MMSE) [20]; depressive symptoms were assessed with the Geriatric Depression Scale (GDS) in its short form [21]; the ability to perform activities of daily living (ADL) [22] and instrumental activities of daily living (IADL) [23] was also assessed. The Cumulative Illness Rating Scale for geriatrics (CIRS-G) [24] was administered to account for comorbidity burden. There were no statistically significant differences between both groups in age, gender, living alone rate, education years, ADL score, MMSE score, number of chronic medications and serum cretinine levels, although higher IADL score, GDS score and CIRS-G total score (though not higher severity index) were observed among the excluded study participants.

\section{Assessment of sarcopenia}

Following the revised EWGSOP2 criteria for an operational definition of sarcopenia [12], all three components, i.e. muscle strength, muscle mass, and physical performance were assessed. Probable sarcopenia was identified when low muscle strength was present, diagnosis of sarcopenia was confirmed when low muscle strength and low muscle mass were both evidenced, and criteria for severe sarcopenia were met when sarcopenia concurred with low physical performance.

Muscle strength was assessed through the handgrip strength test [25], using a hydraulic grip strength dynamometer (Model J00105 JAMAR Hydraulic Hand, Lafayette Instrument Company, USA). Participants were encouraged to squeeze as hard as they could, 3 attempts were allowed for each hand alternating sides and the maximum measurement was registered. EWGSOP2 recommended cut-off points for low muscle strength were used, $<27 \mathrm{~kg}$ for men and $<16 \mathrm{~kg}$ for women.

Body composition in terms of fat and fat-free mass was assessed by BIA using the AKERN BIA $101 \mathrm{New}$ Edition $50 \mathrm{kHz}$ monofrequency device (AKERN SRL, Florence, Italy). Appendicular skeletal muscle mass (ASM) was estimated using the Sergi et al. equation [26], a cross-validated equation for standardisation specifically derived from older European populations, as recommended by the EWGSOP2 consensus. A decision was made to apply no adjustment for body size to ASM measures, as also contemplated in the consensus. Following the EWGSOP2 cut-off points, low muscle mass was defined by an ASM $<20.0 \mathrm{~kg}$ for men and $<15 \mathrm{~kg}$ for women.

Physical performance was assessed by the SPPB, a composite test consisting of a balance test (ability to stand for $10 \mathrm{~s}$ with feet close together side by side, then in semi-tandem and then in full-tandem positions), a gait speed assessment (usual time to walk $4 \mathrm{~m}$ ), and a chair stand test (time to raise from a chair and return to the seated position 5 times without using arms) [27]. A score from 0 to 4 was assigned to each test, thus summing up to a maximum total score of 12 . As suggested by the EWGSOP2 consensus, a total score of $\leq 8$ was considered to indicate low physical performance.

\section{Assessment of kidney function}

Serum creatinine was measured at local level by standard methods. Creatinine-based eGFR was calculated using the following equations:

Berlin Initiative Study (BIS) [28]:

$$
3736 \times \text { creatinine } e^{-0.87} \times \text { age } e^{-0.95} \times 0.82 \text { (if female) }
$$

Chronic Kidney Disease Epidemiological Collaboration (CKD-EPI) [29]: 
Female

$(\mathrm{Scr} \leq 0.7)$

$(\mathrm{Scr}>0.7)$

Male

$(\mathrm{Scr} \leq 0.9) \quad$ eGFR $=141 \times(\mathrm{Scr} / 0.9)^{-0.411} \times(0.993)^{\text {Age }}$

$($ Scr $>0.9) \quad$ eGFR $=141 \times(\text { Scr } / 0.9)^{-1.209} \times(0.993)^{\text {Age }}$

Full Age Spectrum (FAS) [30]:

$[107.3 /($ creatinine $/ Q)] \times 0.988^{(\text {Age-40) }}$ for age $>40$ years

$\mathrm{Q}=$ median Scr value for age-/sex-specific healthy populations.

Categories of CKD were defined according to Kidney Disease Improving Global Outcomes (KDIGO) guidelines [31], moreover CKD categories were we further combined into two groups, i.e., eGFR $\geq 60 \mathrm{ml} / \mathrm{min} / 1.73$ $\mathrm{m}^{2}$ (categories 1 and 2) and eGFR $<60 \mathrm{ml} / \mathrm{min} / 1.73 \mathrm{~m}^{2}$ (categories 3a, 3b and 4). Albumin in urine was detected by urine spot analysis and expressed as mg albumin per gram urine $(\mathrm{mg} / \mathrm{g})$, and albumin-to-creatinine ratio (ACR) was calculated and reported as $\mathrm{mg}$ albumin per gram creatinine $(\mathrm{mg} / \mathrm{g})$. Categories of albuminuria were also defined according to KDIGO guidelines, thus, normoalbuminuria was defined as ACR $<30 \mathrm{mg} / \mathrm{g}$, microalbuminuria as ACR $30-300 \mathrm{mg} / \mathrm{g}$ and macroalbuminuria as $\mathrm{ACR}>300 \mathrm{mg} / \mathrm{g}$.

\section{Statistical analysis}

All variables were checked for normality by the Kolmogorov-Smirnov test. Continuous and normally variables are expressed as mean and standard deviation. Non-normally distributed variables are expressed as median and interquartile difference. Categorical variables are expressed as number and percentage.

The association between categorical variables was analyzed by the Chi-square test, with the correction of continuity when indicated. The relation between quantitative variables, according to sarcopenia categories, was performed by ANOVA test. Statistical significance was set at $p<0.05$. All statistical analyses were performed with SPSS version 24 (SPSS Inc., Chicago, IL, USA) and MedCalc (JMP ${ }^{\circledast}$ statistics software, USA).

\section{Results}

General characteristics of the 1420 participants evaluated are presented in Table 1. Median age was 79.5 years (77.0-83.0), there were 804 (56.6\%) women and 337 participants $(23.7 \%)$ were living alone. Regarding daily life activities, median ADL score was 0.0 (0.0-1.0), and 55 participants (3.9\%) were considered as dependent; median IADL score was $2.0(0.0-8.0)$, and 590 participants

(41.5\%) were considered as dependent. With respect to cognition, median MMSE score was 29.0 (27.0-30.0), with 104 participants (7.3\%) showing MMSE scores < 24 . Regarding depressive symptoms, median GDS score was 2.0 (1.0-4.0), and 170 participants (12\%) exhibited scores of 5 and higher. The median CIRS-G comorbitidy total score was 7.0 (5.0-11.0), while median CIRS-G severity index was $1.5(1.2-1.8)$. The median number of current medications was $6.0(4.0-9.0)$.

Median BMI was $27.0 \mathrm{~kg} / \mathrm{m}^{2}(24.4-30.0)$ and according to a BMI cut-off value of $\geq 30 \mathrm{~kg} / \mathrm{m}^{2}, 359$ participants (25.3\%) were obese, 143 men (23.2\%) and 216 women (26.9\%); whereas according to fat mass percentage (FM\%) cut-off values of $\geq 25 \%$ for men and $\geq 35 \%$ for women, 768 participants (54.1\%) were obese, 355 men (57.6\%) and 413 women (51.4\%), median FM\% was 31.0 (24.9-37.1).

\section{Kidney function and albuminuria}

The median serum creatinine concentration was 0.93 mg/dl (0.78-1.21) (Table 1). Median eGFR values (ml/ $\mathrm{min} / 1.73 \mathrm{~m}^{2}$ ) according to three different equations were 56.4 (45.8-64.8) (BIS), 68.4 (52.6-80.7) (CKD-EPI) and 56.8 (44.7-66.5) (FAS). Distribution of participants among eGFR categories and according to the different equations is presented in Table 1 . When considered together, 873 participants (61.5\%) according to BIS, 494 (34.8\%) according to CKD-EPI, and 841 (59.2\%) according to FAS had stage 3a, 3b or 4 CKD (eGFR $<60 \mathrm{ml} /$ $\left.\mathrm{min} / 1.73 \mathrm{~m}^{2}\right)$. The median ACR value was $12.6 \mathrm{mg} / \mathrm{g}$ (4.7-32.8), and 1037 participants (73\%) had normoalbuminuria, 288 (20.3\%) had microalbuminuria and 95 (6.7\%) had macroalbuminuria.

\section{Sarcopenia components and categories (Table 2)}

Median grip strength was $24.0 \mathrm{~kg}$ (18.0-31.0), and 306 (21.5\%) participants showed low muscle strength according to the aforementioned cut-off values, 136 men $(22.1 \%)$ and 170 women $(21.1 \%) \quad(p=0.672)$. Median ASM as derived from BIA was $18.1 \mathrm{~kg}(15.2-21.7)$, and following the EWGSOP2 recommended cut-off values, 463 participants (32.6\%) exhibited low muscle mass, with a statistically significant female predominance: 312 women $(38.8 \%)$ vs. 151 men $(24.5 \%)(p<0.001)$. Regarding SPPB, median score was 10.0 (8.0-11.0), and considering the recommended cut-off value of $\leq 8$ points, 440 participants (31\%) had low physical performance, a condition also exhibited more often by female participants ( $p=0.003)$ : 275 women (34.2\%) vs. 165 men (26.8\%).

Nearly half of the study sample (599 participants, $42.2 \%)$ showed no derangement in either muscle strength, muscle mass or physical performance, a condition observed in 303 men (49.2\%) and 296 women (26.8\%). Prevalence of sarcopenia in the present study 
Table 1 General characteristics of the study population

\begin{tabular}{|c|c|}
\hline & $N=1420$ \\
\hline Age, years & $79.5(77.0-83.0)$ \\
\hline Women & $804(56.6 \%)$ \\
\hline Living alone & $337(23.7 \%)$ \\
\hline Education, years & $11.0(8.0-14.0)$ \\
\hline ADL score & $0.0(0.0-1.0)$ \\
\hline ADL dependent & $55(3.9 \%)$ \\
\hline IADL score & $2.0(0.0-8.0)$ \\
\hline IADL dependent & $590(41.5 \%)$ \\
\hline MMSE score & $29.0(27.0-30.0)$ \\
\hline MMSE $<24$ & $104(7.3 \%)$ \\
\hline GDS score & $2.0(1.0-4.0)$ \\
\hline GDS > 5 & $170(12.0 \%)$ \\
\hline CIRS-G total score & $7.0(5.0-11.0)$ \\
\hline CIRS-G severity index & $1.5(1.2-1.8)$ \\
\hline Number of current medications & $6.0(4.0-9.0)$ \\
\hline Creatinine, mg/dl & $0.93(0.78-1.21)$ \\
\hline BIS eGFR, ml/min/1.73 $\mathrm{m}^{2}$ & $56.4(45.8-64.8)$ \\
\hline$\geq 90$ & $9(0.6 \%)$ \\
\hline $60-89$ & $538(37.9 \%)$ \\
\hline $45-59$ & $542(38.2 \%)$ \\
\hline $30-44$ & $259(18.2 \%)$ \\
\hline $15-29$ & $72(5.1 \%)$ \\
\hline$\geq 60$ & $547(38.5 \%)$ \\
\hline$<60$ & $873(61.5 \%)$ \\
\hline CKD-EPI eGFR, $\mathrm{ml} / \mathrm{min} / 1.73 \mathrm{~m}^{2}$ & $68.4(52.6-80.7)$ \\
\hline$\geq 90$ & $42(3.0 \%)$ \\
\hline $60-89$ & $884(62.3 \%)$ \\
\hline $45-59$ & $247(17.4 \%)$ \\
\hline $30-44$ & $163(11.5 \%)$ \\
\hline $15-29$ & $84(5.9 \%)$ \\
\hline$\geq 60$ & $926(65.2 \%)$ \\
\hline$<60$ & $494(34.8 \%)$ \\
\hline FAS eGFR, $\mathrm{ml} / \mathrm{min} / 1.73 \mathrm{~m}^{2}$ & $56.8(44.7-66.5)$ \\
\hline$\geq 90$ & $26(1.8 \%)$ \\
\hline $60-89$ & 553 (38.9\%) \\
\hline $45-59$ & 480 (33.8\%) \\
\hline $30-44$ & $253(17.8 \%)$ \\
\hline $15-29$ & 108 (7.6\%) \\
\hline$\geq 60$ & 579 (40.8\%) \\
\hline$<60$ & 841 (59.2\%) \\
\hline $\mathrm{ACR}, \mathrm{mg} / \mathrm{g}$ & $12.6(4.7-32.8)$ \\
\hline$<30$ & 1037 (73.0\%) \\
\hline $30-300$ & 288 (20.3\%) \\
\hline$>300$ & 95 (6.7\%) \\
\hline
\end{tabular}

Table 1 General characteristics of the study population (Continued)

\begin{tabular}{ll}
\hline & $N=1420$ \\
\hline $\begin{array}{l}\text { Anthropometric measurements } \\
\text { height, cm }\end{array}$ & $162.0(156.0-170.0)$ \\
weight, kg & $72.0(62.7-82.0)$ \\
BMl, $\mathrm{kg} / \mathrm{m}^{2}$ & $27.0(24.4-30.0)$ \\
BIA parameters & \\
FFM, percentage & $69.0(62.9-75.0)$ \\
FFM, kg & $48.5(42.6-57.5)$ \\
FFMl, kg/m ${ }^{2}$ & $18.5(17.1-20.2)$ \\
FM, percentage & $31.0(24.9-37.1)$ \\
FM, kg & $21.9(16.7-28.6)$ \\
FMl, kg/m ${ }^{2}$ & $8.3(6.3-10.9)$ \\
ASM, percentage & $0.25(0.23-0.28)$ \\
ASM, kg & $18.1(15.2-21.7)$ \\
ASMl, kg/m ${ }^{2}$ & $6.9(6.2-7.6)$ \\
Grip strength, kg & $24.0(18.0-31.0)$ \\
SPPB score & $10.0(8.0-11.0)$ \\
\hline
\end{tabular}

Note: continuous variables are expressed as median and interquartile difference. Abbreviations ACR Albumin-to-creatinine ratio, $A D L$ Activities of daily living, ASM Appendicular skeletal muscle mass, ASMI Appendicular skeletal muscle mass index, BIA Bioelectrical impedance analysis, BIS Berlin Initiative Study, BMI Body mass index, CIRS-G Cumulative illness rating scale for geriatrics, CKD-EPI Chronic Kidney Disease Epidemiological Collaboration, eGFR Estimated glomerular filtration rate, FAS Full Age Spectrum, FFM Fat-free mass, FFMI Fat-free mass index, FM Fat mass, FMI Fat mass index, GDS Geriatric depression scale, IADL Instrumental activities of daily living, MMSE Mini mental state examination, SSPB Short physical performance battery

was $10.6 \%$, with a total of 150 participants meeting criteria for sarcopenia, 59 men (9.6\%) and 91 women (11.3\%) $(p=0.29)$. Among the non-sarcopenic participants, 1114 (78.5\% of the study sample) had normal muscle strength, 480 men (77.9\%) and 634 women (78.9\%); and 156 participants (11\%) had low muscle strength, 77 men (12.5\%) and 79 women (9.8\%). Among the sarcopenic participants, 65 (4.6\% of the study sample) had normal physical performance, 26 men (4.2\%) and 39 women (4.9\%); and 85 participants $(6 \%)$ had low physical performance, 33 men (5.4\%) and 52 women (6.5\%), therefore meeting criteria for severe sarcopenia. Differences in rates of sarcopenia categories between men and women showed no statistical significance.

Sarcopenia according to kidney function (Tables 3, 4, 5 and 6) According to BIS (Table 3), sarcopenia was significantly more prevalent in the more advanced stages of CKD: $9.6 \%$ in stages 1 and $2\left(e G F R \geq 60 \mathrm{ml} / \mathrm{min} / 1.73 \mathrm{~m}^{2}\right)$ vs. $13.9 \%$ in stages $3 \mathrm{a}, 3 \mathrm{~b}$ and $4\left(\mathrm{eGFR}<60 \mathrm{ml} / \mathrm{min} / 1.73 \mathrm{~m}^{2}\right)(p=$ $0.024)$. Similarly, rates of severe sarcopenia were also higher in participants in CKD stages 3-4 than in CKD stages $1-2$ (4.7\% vs. $10.3 \%)$, with statistical significance $(p=0.005)$, and were highest in CKD stage $4(13.9 \%)$. 
Table 2 Summary of sarcopenia components and sarcopenia categories, stratified by gender

\begin{tabular}{|c|c|c|c|c|}
\hline & Total $(n=1420)$ & Men $(n=616)$ & Women $(n=804)$ & $p$-value \\
\hline \multicolumn{5}{|l|}{ Sarcopenia components } \\
\hline Low muscle strength & $306(21.5 \%)$ & $136(22.1 \%)$ & $170(21.1 \%)$ & 0.672 \\
\hline Low muscle mass & $463(32.6 \%)$ & $151(24.5 \%)$ & $312(38.8 \%)$ & $<0.001$ \\
\hline Low physical performance & $440(31.0 \%)$ & $165(26.8 \%)$ & $275(34.2 \%)$ & 0.003 \\
\hline \multicolumn{5}{|l|}{ Sarcopenia categories } \\
\hline No sarcopenia & $1114(78.5 \%)$ & $480(77.9 \%)$ & $634(78.9 \%)$ & 0.672 \\
\hline Probable sarcopenia & $156(11.0 \%)$ & 77 (12.5\%) & 79 (9.8\%) & 0.110 \\
\hline Confirmed sarcopenia & $65(4.6 \%)$ & $26(4.2 \%)$ & $39(4.9 \%)$ & 0.573 \\
\hline Severe sarcopenia & $85(6.0 \%)$ & $33(5.4 \%)$ & $52(6.5 \%)$ & 0.382 \\
\hline
\end{tabular}

Sarcopenia prevalence was also higher in CKD stages 3-4 compared with CKD stages 1-2 $(p=0.042)$ when eGFR was estimated using CKD-EPI equation (Table 4), although prevalence rates differed slightly: $9.8 \%$ vs. $14.2 \%$. Rates of severe sarcopenia were $5.2 \%$ vs. $9.7 \%$ respectively, though difference was not statistically significant $(p=0.105)$, with rates being lowest in CKD stage 1 (4.8\%), and highest in CKD stage 4 (10.7\%).

Finally, when eGFR was calculated with FAS formula (Table 5), prevalence of sarcopenia was $9.8 \%$ (eGFR $\geq 60$ $\mathrm{ml} / \mathrm{min} / 1.73 \mathrm{~m}^{2}$ ) vs. $12.7 \%$ (eGFR $<60$ ), though differences were not statistically significant $(p=0.119)$. Higher percentages of severely sarcopenic participants were observed among CKD stages 3-4 (9.4\%) compared with stages $1-2(4.8 \%)(\mathrm{p}=0.005)$, and were highest among CKD stage 4 participants (13.9\%).

Therefore, higher rates of sarcopenia and severe sarcopenia were observed among participants in more advanced stages of CKD, irrespective of the equation used for the estimation of eGFR, although sarcopenia prevalence varied slightly in those with eGFR $<60 \mathrm{ml} /$ $\min / 1.73 \mathrm{~m}^{2}: 13.9 \%$ with BIS, $14.2 \%$ with CKD-EPI and $12.7 \%$ with FAS equations.

Finally, the distribution of participants according to ACR categories also yielded significantly higher prevalence rates of sarcopenia with increasing albuminuria categories: $9.3 \%$ in normoalbuminuria, $13.2 \%$ in microalbuminuria and $16.8 \%$ in macroalbuminuria $(p=0.019)$. Similarly, as ACR rised, higher rates of severe sarcopenia were observed: $4.8,8.3$ and $11.6 \%$ respectively, although without statistical significance $(p=0.297)$.

\section{Discussion}

In the SCOPE study, where community-dwelling older adults ( 75 years and older) from 7 European countries within a wide range of kidney function were evaluated

Table 3 Prevalence of sarcopenia and sarcopenia categories according to BIS eGFR $\left(\mathrm{ml} / \mathrm{min} / 1.73 \mathrm{~m}^{2}\right)$ categories

\begin{tabular}{|c|c|c|c|c|c|}
\hline \multirow[b]{2}{*}{ BIS eGFR category } & \multicolumn{2}{|l|}{ Non-sarcopenic $(n=1270)$} & \multicolumn{2}{|l|}{ Sarcopenic $(n=150)$} & \multirow[t]{2}{*}{$p$-value } \\
\hline & No sarcopenia $(n=1114)$ & Probable sarcopenia $(n=156)$ & Confirmed sarcopenia $(n=65)$ & Severe sarcopenia $(n=85)$ & \\
\hline \multirow[t]{2}{*}{$\geq 90(n=9)$} & $6(66.7 \%)$ & & $3(33.3 \%)$ & & \multirow[t]{2}{*}{0.026} \\
\hline & $3(33.3 \%)$ & $3(33.3 \%)$ & $2(22.2 \%)$ & $1(11.1 \%)$ & \\
\hline \multirow[t]{2}{*}{$60-89(n=538)$} & $483(89.8 \%)$ & & $55(10.2 \%)$ & & \multirow[t]{2}{*}{0.745} \\
\hline & $440(81.8 \%)$ & $43(8.0 \%)$ & $26(4.8 \%)$ & $29(5.4 \%)$ & \\
\hline \multirow[t]{2}{*}{$45-59(n=542)$} & $496(91.5 \%)$ & & $46(8.5 \%)$ & & \multirow[t]{2}{*}{0.046} \\
\hline & $436(80.4 \%)$ & $60(11.1 \%)$ & $25(4.6 \%)$ & $21(3.9 \%)$ & \\
\hline \multirow[t]{2}{*}{$30-44(n=259)$} & $225(86.9 \%)$ & & $34(13.1 \%)$ & & \multirow[t]{2}{*}{0.138} \\
\hline & $188(72.6 \%)$ & $37(14.3 \%)$ & $10(3.9 \%)$ & $24(9.3 \%)$ & \\
\hline \multirow[t]{2}{*}{$15-29(n=72)$} & $60(83.3 \%)$ & & $12(16.7 \%)$ & & \multirow[t]{2}{*}{0.084} \\
\hline & $47(65.3 \%)$ & $13(18.1 \%)$ & $2(2.8 \%)$ & $10(13.9 \%)$ & \\
\hline \multirow[t]{2}{*}{$\geq 60(n=1089)$} & 985 (90.4\%) & & $104(9.6 \%)$ & & \multirow[t]{4}{*}{0.024} \\
\hline & 879 (80.7\%) & $106(9.7 \%)$ & $53(4.9 \%)$ & $51(4.7 \%)$ & \\
\hline \multirow[t]{2}{*}{$<60(n=331)$} & $285(86.1 \%)$ & & $46(13.9 \%)$ & & \\
\hline & 235 (71.0\%) & 50 (15.1\%) & 12 (3.6\%) & 34 (10.3\%) & \\
\hline
\end{tabular}


Table 4 Prevalence of sarcopenia and sarcopenia categories according to CKD-EPI eGFR (ml/min/1.73m²) categories

\begin{tabular}{|c|c|c|c|c|c|}
\hline \multirow[b]{2}{*}{$\begin{array}{l}\text { CKD-EPI eGFR } \\
\text { category }\end{array}$} & \multicolumn{2}{|l|}{ Non-sarcopenic $(n=1270)$} & \multicolumn{2}{|l|}{ Sarcopenic $(n=150)$} & \multirow[t]{2}{*}{$p$-value } \\
\hline & No sarcopenia $(n=1114)$ & Probable sarcopenia $(n=156)$ & Confirmed sarcopenia $(n=65)$ & Severe sarcopenia $(n=85)$ & \\
\hline \multirow[t]{2}{*}{$\geq 90(n=42)$} & $37(88.1 \%)$ & & $5(11.9 \%)$ & & \multirow[t]{2}{*}{0.774} \\
\hline & $29(69.0 \%)$ & $8(19.0 \%)$ & $3(7.1 \%)$ & $2(4.8 \%)$ & \\
\hline \multirow[t]{2}{*}{$60-89(n=884)$} & $795(89.9 \%)$ & & $89(10.1 \%)$ & & \multirow[t]{2}{*}{0.435} \\
\hline & $720(81.4 \%)$ & $75(8.5 \%)$ & $46(5.2 \%)$ & $43(4.9 \%)$ & \\
\hline \multirow[t]{2}{*}{$45-59(n=247)$} & $226(91.5 \%)$ & & $21(8.5 \%)$ & & \multirow[t]{2}{*}{0.246} \\
\hline & $194(78.5 \%)$ & $32(13.0 \%)$ & $5(2.0 \%)$ & $16(6.5 \%)$ & \\
\hline \multirow[t]{2}{*}{$30-44(n=163)$} & $139(85.3 \%)$ & & $24(14.7 \%)$ & & \multirow[t]{2}{*}{0.066} \\
\hline & $112(68.7 \%)$ & $27(16.6 \%)$ & $9(5.5 \%)$ & $15(9.2 \%)$ & \\
\hline \multirow[t]{2}{*}{$15-29(n=84)$} & $73(86.9 \%)$ & & $11(13.1 \%)$ & & \multirow[t]{2}{*}{0.436} \\
\hline & $59(70.2 \%)$ & $14(16.7 \%)$ & $2(2.4 \%)$ & $9(10.7 \%)$ & \\
\hline \multirow[t]{2}{*}{$\geq 60(n=1173)$} & $1058(90.2 \%)$ & & 115 (9.8\%) & & \multirow[t]{2}{*}{0.042} \\
\hline & $943(80.4 \%)$ & $115(9.8 \%)$ & $54(4.6 \%)$ & $61(5.2 \%)$ & \\
\hline \multirow[t]{2}{*}{$<60(n=247)$} & $212(85.8 \%)$ & & $35(14.2 \%)$ & & \\
\hline & 171 (69.2\%) & 41 (16.6\%) & 11 (4.5\%) & 24 (9.7\%) & \\
\hline
\end{tabular}

CKD-EPI Chronic Kidney Disease Epidemiological Collaboration, eGFR Estimated glomerular filtration rate

(from normal to stage $4 \mathrm{CKD}$, therefore excluding patients with ESRD); one of ten community-dwelling older adults had sarcopenia, and $6 \%$ of the evaluated participants had severe sarcopenia. Women had higher rates of low muscle mass and more often exhibited low physical performance than men; and although sarcopenia was present in $11.3 \%$ of women and $9.6 \%$ of men, and severe sarcopenia in $6.5 \%$ of women and $5.4 \%$ of men, differences were not statistically significant. Our study found that a higher prevalence of sarcopenia is observed among participants with poorer kidney function categories (CKD stages 3-4) compared with participants with better kidney function categories (CKD stages 1-2), irrespective of the equation used to estimate eGFR (BIS, CKD-EPI or FAS), with prevalence rates of 13.9, 14.2 and $12.7 \%$ respectively, according to the EWGSOP2 revised criteria for sarcopenia [12]. These findings are clinically relevant, especially when considering that sarcopenia may affect mobility and increase the risk of falls among older individuals. Indeed, muscle mass and strength were formerly found to be

Table 5 Prevalence of sarcopenia and sarcopenia categories according to FAS eGFR $\left(\mathrm{ml} / \mathrm{min} / 1.73 \mathrm{~m}^{2}\right)$ categories

\begin{tabular}{|c|c|c|c|c|c|}
\hline \multirow[b]{2}{*}{ FAS eGFR category } & \multicolumn{2}{|l|}{ Non-sarcopenic $(n=1270)$} & \multicolumn{2}{|l|}{ Sarcopenic $(n=150)$} & \multirow[t]{2}{*}{$p$-value } \\
\hline & No sarcopenia $(n=1114)$ & Probable sarcopenia $(n=156)$ & Confirmed sarcopenia $(n=65)$ & Severe sarcopenia $(n=85)$ & \\
\hline \multirow[t]{2}{*}{$\geq 90(n=26)$} & $22(84.6 \%)$ & & $4(15.4 \%)$ & & \multirow[t]{2}{*}{0.420} \\
\hline & $16(61.5 \%)$ & $6(23.1 \%)$ & $2(7.7 \%)$ & $2(7.7 \%)$ & \\
\hline \multirow[t]{2}{*}{$60-89(n=553)$} & $496(89.7 \%)$ & & $57(10.3 \%)$ & & \multirow[t]{2}{*}{0.802} \\
\hline & $456(82.5 \%)$ & $40(7.2 \%)$ & $29(5.2 \%)$ & $28(5.1 \%)$ & \\
\hline \multirow[t]{2}{*}{$45-59(n=480)$} & 437 (91.0\%) & & $43(9.0 \%)$ & & \multirow[t]{2}{*}{0.160} \\
\hline & $380(79.2 \%)$ & $57(11.9 \%)$ & $22(4.6 \%)$ & $21(4.4 \%)$ & \\
\hline \multirow[t]{2}{*}{$30-44(n=253)$} & $225(89.0 \%)$ & & $28(11.1 \%)$ & & \multirow[t]{2}{*}{0.774} \\
\hline & $188(74.3 \%)$ & $37(14.6 \%)$ & $9(3.6 \%)$ & $19(7.5 \%)$ & \\
\hline \multirow[t]{2}{*}{$15-29(n=108)$} & $90(83.3 \%)$ & & $18(16.7 \%)$ & & \multirow[t]{2}{*}{0.032} \\
\hline & $74(68.5 \%)$ & $16(14.8 \%)$ & $3(2.8 \%)$ & $15(13.9 \%)$ & \\
\hline \multirow[t]{2}{*}{$\geq 60(n=1059)$} & $955(90.2 \%)$ & & $104(9.8 \%)$ & & \multirow[t]{2}{*}{0.119} \\
\hline & $852(80.5 \%)$ & $103(9.7 \%)$ & $53(5.0 \%)$ & $51(4.8 \%)$ & \\
\hline \multirow[t]{2}{*}{$<60(n=361)$} & $315(87.3 \%)$ & & $46(12.7 \%)$ & & \\
\hline & $262(72.6 \%)$ & $53(14.7 \%)$ & $12(3.3 \%)$ & $34(9.4 \%)$ & \\
\hline
\end{tabular}


Table 6 Prevalence of sarcopenia and sarcopenia categories according to ACR $(\mathrm{mg} / \mathrm{g})$ categories

\begin{tabular}{|c|c|c|c|c|c|}
\hline \multirow[b]{2}{*}{ ACR category } & \multicolumn{2}{|l|}{ Non-1sarcopenic $(n=1270)$} & \multicolumn{2}{|l|}{ Sarcopenic $(n=150)$} & \multirow[t]{2}{*}{$p$-value } \\
\hline & No sarcopenia $(n=1114)$ & Probable sarcopenia $(n=156)$ & Confirmed sarcopenia $(n=65)$ & Severe sarcopenia $(n=85)$ & \\
\hline \multirow[t]{2}{*}{$<30(n=1037)$} & $941(90.7 \%)$ & & $96(9.3 \%)$ & & \multirow[t]{2}{*}{0.008} \\
\hline & $842(81.2 \%)$ & 99 (9.5\%) & $46(4.4 \%)$ & $50(4.8 \%)$ & \\
\hline \multirow[t]{2}{*}{$30-300(n=288)$} & $250(86.8 \%)$ & & $38(13.2 \%)$ & & \multirow[t]{2}{*}{0.104} \\
\hline & $211(73.3 \%)$ & 39 (13.5\%) & $14(4.9 \%)$ & $24(8.3 \%)$ & \\
\hline \multirow[t]{2}{*}{$>300(n=95)$} & 79 (83.2\%) & & $16(16.8 \%)$ & & \multirow[t]{2}{*}{0.039} \\
\hline & 61 (64.2\%) & $18(18.9 \%)$ & $5(5.3 \%)$ & $11(11.6 \%)$ & \\
\hline
\end{tabular}

$A C R$ Albumin-to-creatinine ratio

linearly associated with mobility impairment [32], and the contribution of sarcopenia to unexplained falls is still to be elucidated [33]. Thus, our results suggest that the assessment of sarcopenia may be helpful in identifying older CKD patients at risk of falling and implementing inherent preventive measures.

Previous studies have found differences in prevalence estimates of sarcopenia when operational criteria proposed from various existing consensus were compared [9-11]. A recent systematic review and meta-analysis [10] found higher prevalence rates when a single measure of muscle mass was used to define sarcopenia instead of composite definitions, and that prevalence estimates also depended on the use of BIA vs. dualenergy X-ray absorptiometry (DXA) to assess muscle mass, the cut-off points employed or even the method of adjustment for body size. Recently, EWGSOP2 criteria have been found to yield lower prevalence estimates (9.3\% overall, $11.9 \%$ in men and $6.7 \%$ in women) than EWGSOP criteria $(20.8 \%$ overall, $25.5 \%$ in men and $16.2 \%$ in women) [11]. The combination of different tools and adjustments also impacted prevalence rates; specifically, the use of grip strength and ASM to define sarcopenia yielded a prevalence of $11.7 \%$, resembling that observed in the SCOPE study though differing on the gender predominance; likewise, the use of grip strength, ASM and SPPB to define severe sarcopenia yielded a prevalence of $2.2 \%$. Other recent studies in community-dwelling older adults have used EWGSOP2 criteria $[34,35]$ with varying prevalence rates of sarcopenia (20 and 3.4\% respectively) and severe sarcopenia $(1.8 \%$ vs. $3.2 \%)$, though different assessment methods were employed.

Sarcopenia has been reported to be common in community-dwelling adults with CKD, with prevalence rising markedly with declining kidney function [36]. Specifically, in adults with eGFR $\geq 90$, eGFR $60-89$ and eGFR $<60 \mathrm{ml} / \mathrm{min} / 1.73 \mathrm{~m}^{2}$ prevalence was $26.6,38.9$, and $60.1 \%$ respectively. It is worth noting that only muscle mass with a less restringing cut-off was employed to define sarcopenia, which could account for the higher prevalence observed as compared with our results. Sarcopenia has been found to be highly prevalent among patients with CKD [37], and more prevalent among persons with lower eGFR, with stage 4 CKD being independently associated with an increased likelihood of sarcopenia. CKD has been reported to be a major risk factor of sarcopenia in community-dwelling older men [38], and that even stage $3 \mathrm{CKD}$ had a more than threefold risk of skeletal muscle mass (SMM) reduction.

In patients with stages 3-5 CKD on conservative therapy, prevalence of sarcopenia was found to vary according to the method used to assess muscle mass, with BIA derived SMMI yielding lower estimates (5.9\%) as opposed to the use of midarm muscle circumference $(9.8 \%)$ or subjective global assessment $(9.4 \%)$ as surrogates of muscle mass [39]. Noteworthy, this study assessed both muscle mass and strength components of sarcopenia, which could account for the lower prevalence rates observed, far more similar to our results. Another study in a Korean population [40] found that prevalence of sarcopenia was higher in patients even with early stage kidney disease, with prevalence raising as the stage of CKD increased from eGFR $\geq 90$, to eGFR 60-89.9, and eGFR $<60 \mathrm{ml} / \mathrm{min} / 1.73 \mathrm{~m}^{2}(2.6,5.6$ and $18.1 \%$ respectively in men, and $5.3,7.1$ and $12.6 \%$ respectively in women), although a statistically significant association between sarcopenia and stage of CKD was found in men but not in women. In this regard, our study found a higher prevalence among women across virtually all CKD stages. Other studies in CKD patients have observed variable prevalence rates of sarcopenia: $34.5 \%$ (stages 2-3a) and 65.5\% (stages 3b-5) [14]; 37\% (stages 3-5) [41]; 14\% (stages 3-5) greater in men (16\%) than in women $(8 \%)$ and with a significant positive relationship between ASM and GFR [15]; and 12.5\% (stages $3 \mathrm{~b}-4$ in men aged $60-74$ ) or $55 \%$ (stages $3 \mathrm{~b}-4$ in men aged $>$ 75) [42].

Although several previous studies have evaluated the relationship between muscle mass and/or muscle strength and kidney function, to our knowledge this is the first study to assess sarcopenia according to CKD stages incorporating the revised EWGSOP2 criteria for its definition. Furthermore, varying methods for estimating 
GFR have been employed, mainly the CKD-EPI and Modification of Diet in Renal Disease (MDRD) equations, whereas few studies have employed measured GFR, e.g., through iohexol clearance method [15]. Sarcopenia is a muscle disease closely related to ageing, and its prevalence increases sharply within ageing populations, in which concern exists about the accuracy of GFR estimating equations. Performance of such equations has been compared against equations specifically developed in older populations with varying results [43]. In the SCOPE study it has been demonstrated that CKD-EPI, BIS and FAS equations cannot be considered interchangeable in a community-dwelling older population, and that muscle mass may represent a major source of discrepancy among equations [data not published]. In the present study, the percentage of individuals with sarcopenia in CKD stages 3-4 varied slightly according to the equations employed: $13.9 \%$ for BIS, $14.2 \%$ for CKD-EPI and $12.7 \%$ for FAS equation.

An increased risk of albuminuria, which is associated with mortality, cardiovascular disease and CKD progression, has been reported in patients with sarcopenia and vice versa, independently of CKD [17]. This association has been found to be particularly strong in the older adult population [44] and synergistic with obesity, and also demonstrated in patients with type 2 diabetes [16]. Furthermore, sarcopenia has been associated with an increased risk of progression of albuminuria in a retrospective study of diabetic patients [45], and with an increased risk of incident albuminuria in a recent prospective study in participants without CKD [46]. Common underlying mechanisms have been suggested to account for this association, such as inflammation, insulin resistance, endothelial dysfunction and reninangiotensin-aldosterone system activation. In the present study a higher prevalence of sarcopenia is observed with increasing ACR category: 9.3\% in normoalbuminuric participants, $13.2 \%$ in the microalbuminuric group and $16.8 \%$ in participants with macroalbuminuria $(p=0.019)$.

Some strengths of the present study are inherent to the SCOPE study design, which included a remarkable number of community-dwelling older adults from different centers across Europe, following highly inclusive criteria to ensure the enrolment of a representative sample of real-world outpatients, within a wide range of kidney function categories. Though previous studies have investigated sarcopenia prevalence, few studies have taken CKD status into account, especially regarding CKD patients not on dialysis. Furthermore, the association of higher rates of sarcopenia and kidney disease may depend not only on CKD stage or degree of eGFR but also on albuminuria status, with fewer studies assessing both variables concomitantly or comparing different eGFR equations. Moreover, as new revised definitions of sarcopenia arise following the increasing knowledge of this condition, the prevalence rates, the association with risk factors or predisposing conditions, and even the association with outcomes may differ from what was previously assumed. Thus, this is the first study to investigate sarcopenia in patients not in ESRD incorporating the newly recommended criteria by the EWGSOP2 consensus for the definition of sarcopenia and its subtypes through its three components (i.e.: muscle mass, muscle strength and physical performance); and also assessing not only different equations for the estimation of GFR, but also albuminuria status as relevant measures related to kidney function.

Some limitations deserve consideration. First, the present study represents a cross-sectional analysis of SCOPE study, and causality in the relationship between sarcopenia and kidney function or albuminuria cannot be established. Second, the exclusion of ESRD participants from the SCOPE study limits its applicability in this group of patients. Third, although participants not included in the analysis because of missing data on any of the sarcopenia components did not seem to differ on the majority of general characteristics from those who completed sarcopenia assessment; a poorer clinical status, poorer comorbidity profile or higher physical disability in those participants could have led to an underestimation of sarcopenia prevalence in our study. Fourth, the use of BIA for the estimation of ASM, although endorsed by different consensuses because of its affordability, availability and portability, may exhibit some limitations as compared with DXA measurements or with magnetic resonance imaging/computed tomography (currently considered as gold standards for noninvasive assessment of muscle mass); and hydration status has to be taken into account as it may influence results, especially in CKD patients. Moreover, concern exists regarding the use of height-adjusted ASM to correct for body size, as it tends to underestimate the prevalence of sarcopenia and may not be suitable for all populations. As EWGSOP2 consensus makes no recommendation to adjust for body size, a decision was made to employ non-adjusted ASM as a measure of muscle mass. Finally the use of noncreatinine based equations for the estimation of GFR, such as eGFR based on cystatin C, might be more accurate for the evaluation of sarcopenia in these patients; thus studies comparing eGFR equations based on cystatin $\mathrm{C}$ or incorporating measured GFR by iohexol clearance may be of paramount relevance. Creatinine is a metabolic product of creatine and phosphocreatine arising from the muscle compartment, which is directly related to muscle mass. Thus, muscle wasting may lead to an overestimation of eGFR in sarcopenic patients, which should be taken into account when evaluating such patients. Further research is needed to investigate the underlying mechanisms of sarcopenia among older adults with CKD and albuminuria. 


\section{Conclusions}

The main finding from our study is the relevant prevalence of sarcopenia observed among communitydwelling European adults aged 75 years and older, using the most recent diagnostic criteria for sarcopenia endorsed by the EWGSOP2 consensus. Participants within poorer eGFR categories, irrespective of the equation used for its calculation, have a higher prevalence of sarcopenia and are more often severely sarcopenic. There are though some differences in prevalence according to the eGFR formula used. Moreover, participants within higher albuminuria categories are more often sarcopenic, with higher rates of severe sarcopenia. Therefore, prompt assessment of sarcopenia status may be warranted in the usual care of older people with impaired kidney function and/or albuminuria, which could allow for its early detection and trigger proper interventions.

\begin{abstract}
Abbreviations
ACR: Albumin-to-creatinine ratio; ADL: Activities of daily living;

ASM: Appendicular skeletal muscle mass; ASMI: Appendicular skeletal muscle mass index; AWGS: Asian Working Group for Sarcopenia; BIA: Bioelectrical impedance analysis; BIS: Berlin Initiative Study; BMI: Body mass index; CGA: Comprehensive geriatric assessment; CIRS-G: Cumulative illness rating scale for geriatrics; CKD: Chronic kidney disease; CKD-EPI: Chronic Kidney Disease Epidemiological Collaboration; DXA: Dual-energy X-ray absorptiometry; eGFR: Estimated glomerular filtration rate; ESPEN: European Society of Clinical Nutrition; ESRD: End-stage renal disease; EWGSOP: European Working Group on Sarcopenia in Older People; FAS: Full Age Spectrum; FFM: Fat-free mass; FFMI: Fat-free mass index; FM: Fat mass; FMI: Fat mass index; FNIH: Foundation for the National Institutes of Health; GDS: Geriatric depression scale; GFR: Glomerular filtration rate; IADL: Instrumental activities of daily living; IWGS: International Working Group on Sarcopenia; KDIGO: Kidney Disease Improving Global Outcomes; MDRD: Modification of Diet in Renal Disease; MMSE: Mini mental state examination; SCOPE: Screening for Chronic Kidney Disease among Older People across Europe; SCWD: Society on Sarcopenia, Cachexia and Wasting Disorders; SMM: Skeletal muscle mass; SMMI: Skeletal muscle mass index; SSPB: Short physical performance battery
\end{abstract}

\section{Acknowledgments}

We thank CERCA Programme / Generalitat de Catalunya and Hospital Universitari de Bellvitge / Institut Català de la Salut for institutional support. SCOPE study investigators.

Coordinating center, Fabrizia Lattanzio, Italian National Research Center on Aging (INRCA), Ancona, Italy - Principal Investigator. Andrea Corsonello, Silvia Bustacchini, Silvia Bolognini, Paola D'Ascoli, Raffaella Moresi, Giuseppina Di Stefano, Cinzia Giammarchi, Anna Rita Bonfigli, Roberta Galeazzi, Federica Lenci, Stefano Della Bella, Enrico Bordoni, Mauro Provinciali, Robertina Giacconi, Cinzia Giuli, Demetrio Postacchini, Sabrina Garasto, Annalisa Cozza Italian National Research Center on Aging (INRCA), Ancona, Fermo and Cosenza, Italy - Coordinating staff. Romano Firmani, Moreno Nacciariti, Mirko Di Rosa, Paolo Fabbietti - Technical and statistical support. Participating centers

- Department of Internal Medicine, Medical University of Graz, Austria: Gerhard Hubert Wirnsberger, Regina Elisabeth Roller-Wirnsberger, Carolin Herzog, Sonja Lindner.

- Section of Geriatric Medicine, Department of Internal Medicine, Erasmus University Medical Center Rotterdam, The Netherlands: Francesco MattaceRaso, Lisanne Tap, Gijsbertus Ziere, Jeannette Goudzwaard.

- Department of Geriatrics, Healthy Ageing Research Centre, Medical University of Lodz, Poland: Tomasz Kostka, Agnieszka Guligowska, Łukasz Kroc, Bartłomiej K Sołtysik, Katarzyna Smyj, Elizaveta Fife, Joanna Kostka, Małgorzata Pigłowska Department of Geriatrics, Healthy Ageing Research Centre, Medical University of Lodz, Poland: Tomasz Kostka, Agnieszka Guligowska, Łukasz Kroc, Bartłomiej K Sołtysik, Małgorzata Pigłowska,
Agnieszka Wójcik, Zuzanna Chrząstek, Natalia Sosowska, Anna Telążka, Joanna Kostka, Katarzyna Smyj.

- The Recanati School for Community Health Professions at the faculty of Health Sciences at Ben-Gurion University of the Negev, Israel: Rada ArtziMedvedik, Yehudit Melzer, Mark Clarfield, Itshak Melzer; and Maccabi Healthcare services southern region, Israel: Rada Artzi-Medvedik, Ilan Yehoshua, Yehudit Melzer

- Geriatric Unit, Internal Medicine Department and Nephrology Department, Hospital Universitari de Bellvitge, Institut d'Investigació Biomèdica de Bellvitge - IDIBELL, L'Hospitalet de Llobregat, Barcelona, Spain: Francesc Formiga, Rafael Moreno-González, Xavier Corbella, Yurema Martínez, Carolina Polo, Josep Maria Cruzado.

- Department of Geriatric Medicine, Hospital Clínico San Carlos, Madrid, Spain: Pedro Gil Gregorio, Sara Laínez Martínez, Monica González Alonso, Jose A. Herrero Calvo, Fernando Tornero Molina, Lara Guardado Fuentes,

Pamela Carrillo García, María Mombiedro Pérez.

- Department of General Internal Medicine and Geriatrics, Krankenhaus Barmherzige Brüder Regensburg and Institute for Biomedicine of Aging, Friedrich-Alexander-Universität Erlangen-Nürnberg, Germany: Christian Weingart, Ellen Freiberger, Cornel Sieber.

- Department of Medical Sciences, Uppsala University, Sweden: Johan Ärnlöv, Axel Carlsson, Tobias Feldreich.

Scientific advisory board (SAB).

Roberto Bernabei, Catholic University of Sacred Heart, Rome, Italy.

Christophe Bula, University of Lausanne, Switzerland.

Hermann Haller, Hannover Medical School, Hannover, Germany.

Carmine Zoccali, CNR-IBIM Clinical Epidemiology and Pathophysiology of

Renal Diseases and Hypertension, Reggio Calabria, Italy.

Data and Ethics Management Board (DEMB).

Dr. Kitty Jager, University of Amsterdam, The Netherlands.

Dr. Wim Van Biesen, University Hospital of Ghent, Belgium.

Paul E. Stevens, East Kent Hospitals University NHS Foundation Trust, Canterbury, United Kingdom. We thank the BioGer IRCCS INRCA Biobank for the collection of the SCOPE samples.

\section{About this supplement}

This article has been published as part of BMC Geriatrics Volume 20 Supplement 1 2020: The Screening for Chronic Kidney Disease among Older People across Europe (SCOPE) project: findings from cross-sectional analysis. The full contents of the supplement are available at https://bmcgeriatr.biomedcentral.com/articles/supplements/volume-20-supplement-1.

\section{Authors' contributions}

RMG, XC and FF participated in study protocol design, data collection and drafting of the manuscript. AC and FL conceived the study, coordinated study protocol and data collection, participated in manuscript revision and approval. PF participated in data analysis, manuscript drafting and revision. $X C, F M R, L T, C S, E F, T K, A G, I M, Y M, A C C, J A ̈, ~ R R W, G W, P G$, SLM, participated in study protocol design, data collection, and manuscript revision and approval. All authors read and approved the final manuscript.

\section{Funding}

SCOPE study and publication costs are funded by the European Union Horizon 2020 program, under the Grant Agreement $n^{\circ}$ 634869. Funding body had no role in the design of the study and collection, analysis, and interpretation of data and in writing the manuscript.

\section{Availability of data and materials}

Data will be available for SCOPE researchers through the project website (www.scopeproject.eu).

\section{Ethics approval and consent to participate}

The study protocol was approved by ethics committees at all participating institutions, and complies with the Declaration of Helsinki and Good Clinical Practice Guidelines. All patients signed a written informed consent to be enrolled. Only baseline data are used in the present study. Ethics approvals have been obtained by Ethics Committees in participating institutions as follows:

Italian National Research Center on Aging (INRCA), Italy, \#2015 0522 IN, January 27, 2016.

University of Lodz, Poland, \#RNN/314/15/KE, November 17, 2015. 
Medizinische Universität Graz, Austria, \#28-314 ex 15/16, August 5, 2016. Erasmus Medical Center Rotterdam, The Netherland, \#MEC-2016-036 \#NL56039.078.15, v.4, March 7, 2016.

Hospital Clínico San Carlos, Madrid, Spain, \# 15/532-E_BC, September 16, 2016.

Bellvitge University Hospital Barcellona, Spain, \#PR204/15, January 29, 2016.

Friedrich-Alexander University Erlangen-Nürnberg, Germany, \#340_15B,

January 21, 2016.

Helsinki committee in Maccabi Healthcare services, Bait Ba-lev, Bat Yam, Israel, \#45/2016, July 24, 2016.

\section{Consent for publication}

Not applicable.

\section{Competing interests}

The authors declare that they have no competing interests.

\section{Author details}

${ }^{1}$ Geriatric Unit, Internal Medicine Department, Hospital Universitari de Bellvitge, Systemic Diseases and Ageing Group, Cardiovascular, Respiratory and Systemic Diseases and Cellular Aging Program, Institut d'Investigació Biomèdica de Bellvitge (IDIBELL), L'Hospitalet de Llobregat, Barcelona, Spain. ${ }^{2}$ Hestia Chair in Integrated Health and Social Care, Faculty of Medicine and Health Sciences, Catalunya International University, Barcelona, Spain. ${ }^{3}$ Department of Internal Medicine, Section of Geriatric Medicine, Erasmus MC, University Medical Center Rotterdam, Rotterdam, The Netherlands. ${ }^{4}$ Department of Internal Medicine-Geriatrics, Institute for Biomedicine of Aging (IBA), Friedrich-Alexander-Universität Erlangen-Nürnberg, Nürnberg, Germany. ${ }^{5}$ Department of Geriatrics, Healthy Ageing Research Centre, Medical University of Lodz, Lodz, Poland. 'Department of Physical Therapy, Recanati School for Community Health Professions at the faculty of Health Sciences, Ben-Gurion University of the Negev, Beer-sheva, Israel. ${ }^{7}$ Maccabi Health Organization, Tel Aviv-Yafo, Israel. ${ }^{8}$ Department of Medical Sciences, Uppsala University, Uppsala, Sweden. ${ }^{9}$ Division of Family Medicine, Department of Neurobiology, Care Sciences and Society, Karolinska Institutet, Stockholm, Sweden. ${ }^{10}$ School of Health and Social Studies, Dalarna University, Falun, Sweden. ${ }^{11}$ Department of Internal Medicine, Medical University of Graz, Graz, Austria. ${ }^{12}$ Geriatric Department, Hospital Clínico San Carlos, Martín Lagos S/N, 28040 Madrid, Spain. ${ }^{13}$ Laboratory of Geriatric Pharmacoepidemiology and Biostatistics, IRCCS INRCA, Via S. Margherita 5, 60124 Ancona, Italy. ${ }^{14}$ Italian National Research Center on Aging (IRCCS INRCA), Ancona, Fermo and Cosenza, Italy.

\section{Received: 5 August 2020 Accepted: 11 August 2020}

\section{Published: 2 October 2020}

\section{References}

1. Cuesta F, Formiga F, Lopez-Soto A, Masanes F, Ruiz D, Artaza I, Salvà A, Serra-Rexach JA, Rojano I, Luque X, Cruz-Jentoft AJ. Prevalence of sarcopenia in patients attending outpatient geriatric clinics: the ELLI study. Age Ageing. 2015;44:807-9. https://doi.org/10.1093/ageing/afv088.

2. Morley JE, Anker SD, von Haehling S. Prevalence, incidence, and clinical impact of sarcopenia: facts, numbers, and epidemiology-update 2014. J Cachexia Sarcopenia Muscle. 2014;5:253-9. https://doi.org/10.1007/s13539014-0161-y.

3. Muscaritoli M, Anker SD, Argilés J, Aversa Z, Bauer JM, Biolo G, Boirie Y, Bosaeus I, Cederholm T, Costelli P, Fearon KC, Laviano A, Maggio M, Rossi Fanelli F, Schneider SM, Schols A, Sieber CC. Consensus definition of sarcopenia, cachexia and pre-cachexia: joint document elaborated by special interest groups (SIG) "cachexia-anorexia in chronic wasting diseases" and "nutrition in geriatrics". Clin Nutr. 2010;29:154-9. https://doi.org/10. 1016/j.clnu.2009.12.004

4. Cruz-Jentoft AJ, Baeyens JP, Bauer JM, Boirie Y, Cederholm T, Landi F, Martin FC, Michel JP, Rolland Y, Schneider SM, Topinková E, Vandewoude M, Zamboni M. European working group on sarcopenia in older people. Sarcopenia: European consensus on definition and diagnosis: report of the European working group on sarcopenia in older people. Age Ageing. 2010; 39:412-23. https://doi.org/10.1093/ageing/afq034.

5. Fielding RA, Vellas B, Evans WJ, Bhasin S, Morley JE, Newman AB, Abellan van Kan G, Andrieu S, Bauer J, Breuille D, Cederholm T, Chandler J, De Meynard C, Donini L, Harris T, Kannt A, Keime Guibert F, Onder G,
Papanicolaou D, Rolland Y, Rooks D, Sieber C, Souhami E, Verlaan S, Zamboni M. Sarcopenia: an undiagnosed condition in older adults. Current consensus definition: prevalence, etiology, and consequences. International working group on sarcopenia. J Am Med Dir Assoc. 2011;12:249-56. https:// doi.org/10.1016/j.jamda.2011.01.003.

6. Morley JE, Abbatecola AM, Argiles JM, Baracos V, Bauer J, Bhasin S, Cederholm T, Coats AJ, Cummings SR, Evans WJ, Fearon K, Ferrucci L, Fielding RA, Guralnik JM, Harris TB, Inui A, Kalantar-Zadeh K, Kirwan BA, Mantovani G, Muscaritoli M, Newman AB, Rossi-Fanelli F, Rosano GM, Roubenoff R, Schambelan M, Sokol GH, Storer TW, Vellas B, von Haehling S, Yeh SS, Anker SD. Society on Sarcopenia, Cachexia and Wasting Disorders Trialist Workshop. Sarcopenia with limited mobility: an international consensus. J Am Med Dir Assoc. 2011;12:403-9. https://doi.org/10.1016/j. jamda.2011.04.014

7. Chen LK, Liu LK, Woo J, Assantachai P, Auyeung TW, Bahyah KS, Chou MY, Chen LY, Hsu PS, Krairit O, Lee JS, Lee WJ, Lee Y, Liang CK, Limpawattana P, Lin CS, Peng LN, Satake S, Suzuki T, Won CW, Wu CH, Wu SN, Zhang T, Zeng P, Akishita M, Arai H. Sarcopenia in Asia: consensus report of the Asian working Group for Sarcopenia. J Am Med Dir Assoc. 2014;15:95-101. https:// doi.org/10.1016/j.jamda.2013.11.025

8. Studenski SA, Peters KW, Alley DE, Cawthon PM, McLean RR, Harris TB, Ferrucci L, Guralnik JM, Fragala MS, Kenny AM, Kiel DP, Kritchevsky SB, Shardell MD, Dam TT, Vassileva MT. The FNIH sarcopenia project: rationale, study description, conference recommendations, and final estimates. Gerontol A Biol Sci Med Sci. 2014;69:547-58. https://doi.org/10.1093/gerona/ glu010.

9. Dam TT, Peters KW, Fragala M, Cawthon PM, Harris TB, McLean R, Shardell M, Alley DE, Kenny A, Ferrucci L, Guralnik J, Kiel DP, Kritchevsky S, Vassileva MT, Studenski S. An evidence-based comparison of operational criteria for the presence of sarcopenia. J Gerontol A Biol Sci Med Sci. 2014;69:584-90. https://doi.org/10.1093/gerona/glu013.

10. Mayhew AJ, Amog K, Phillips S, Parise G, McNicholas PD, de Souza RJ, Thabane L, Raina P. The prevalence of sarcopenia in community-dwelling older adults, an exploration of differences between studies and within definitions: a systematic review and meta-analyses. Age Ageing. 2019;48:4856. https://doi.org/10.1093/ageing/afy106.

11. Kim M, Won CW. Prevalence of sarcopenia in community-dwelling older adults using the definition of the European working group on sarcopenia in older people 2: findings from the Korean frailty and aging cohort study. Age Ageing. 2019;48:910-6. https://doi.org/10.1093/ageing/afz091.

12. Cruz-Jentoft AJ, Bahat G, Bauer J, Boirie $Y$, Bruyère O, Cederholm T, Cooper C, Landi F, Rolland Y, Sayer AA, Schneider SM, Sieber CC, Topinkova E, Vandewoude M, Visser M, Zamboni M, Writing Group for the European Working Group on sarcopenia in older people 2 (EWGSOP2), and the extended group for EWGSOP2. Sarcopenia: revised European consensus on definition and diagnosis. Age Ageing. 2019;48:16-31. https://doi.org/10. 1093/ageing/afz046.

13. Domański M, Ciechanowski K. Sarcopenia: a major challenge in elderly patients with end-stage renal disease. J Aging Res. 2012;2012:754739. https://doi.org/10.1155/2012/754739.

14. Souza VA, Oliveira D, Barbosa SR, Corrêa JODA, Colugnati FAB, Mansur HN, Fernandes NMDS, Bastos MG. Sarcopenia in patients with chronic kidney disease not yet on dialysis: analysis of the prevalence and associated factors. PLoS One. 2017;12:e0176230. https://doi.org/10.1371/journal.pone.0176230.

15. Zhou Y, Hellberg M, Svensson P, Höglund P, Clyne N. Sarcopenia and relationships between muscle mass, measured glomerular filtration rate and physical function in patients with chronic kidney disease stages 3-5. Nephrol Dial Transplant. 2018;33:342-8. https://doi.org/10.1093/ndt/gfw466.

16. Chung HS, Hwang SY, Choi JH, Lee HJ, Yoo HJ, Seo JA, Kim SG, Kim NH, Choi DS, Baik SH, Choi KM. Effects of low muscle mass on albuminuria and chronic kidney disease in patients with type 2 diabetes: the Korean Sarcopenic obesity study (KSOS). J Gerontol A Biol Sci Med Sci. 2018;73:38692. https://doi.org/10.1093/gerona/glx055.

17. Kim TN, Lee EJ, Hong JW, Kim JM, Won JC, Kim MK, Noh JH, Ko KS, Rhee BD, Kim DJ. Relationship Between Sarcopenia and Albuminuria: The 2011 Korea National Health and Nutrition Examination Survey. Medicine (Baltimore). 2016;95:e2500. https://doi.org/10.1097/MD.0000000000002500.

18. Corsonello A, Tap L, Roller-Wirnsberger R, Wirnsberger G, Zoccali C, Kostka T, Guligowska A, Mattace-Raso F, Gil P, Fuentes LG, Meltzer I, Yehoshua I, Formiga-Perez F, Moreno-González R, Weingart C, Freiberger E, Ärnlöv J, Carlsson AC, Bustacchini S, Lattanzio F. SCOPE investigators. Design and 
methodology of the screening for CKD among older patients across Europe (SCOPE) study: a multicenter cohort observational study. BMC Nephrol. 2018;19:260. https://doi.org/10.1186/s12882-018-1030-2.

19. Cederholm T, Barazzoni R, Austin P, Ballmer P, Biolo G, Bischoff SC, Compher C, Correia I, Higashiguchi T, Holst M, Jensen GL, Malone A, Muscaritoli M, Nyulasi I, Pirlich M, Rothenberg E, Schindler K, Schneider SM, de van der Schueren MA, Sieber C, Valentini L, YU JC, Van Gossum A, Singer P. ESPEN guidelines on definitions and terminology of clinical nutrition. Clin Nutr. 2017 Feb;36:49-64. https://doi.org/10.1016/j.cInu.2016.09.004.

20. Folstein MF, Folstein SE, McHugh PR. "mini-mental state". A practical method for grading the cognitive state of patients for the clinician. J Psychiatr Res. 1975 Nov;12(3):189-98.

21. Sheikh JI, Yesavage JA. Geriatric depression scale (GDS): recent evidence and development of a shorter version. Clin Gerontol. 1986;5:165-73.

22. Katz S, Ford AB, Moskowitz RW, Jackson BA, Jaffe MW. Studies of illness in the aged. The index of ADL: a standardized measure of biological and psychosocial function. JAMA. 1963;185:914-9.

23. Lawton MP, Brody EM. Assessment of older people: self-maintaining and instrumental activities of daily living. Gerontologist. 1969:9:179-86.

24. Miller MD, Paradis CF, Houck PR, Mazumdar S, Stack JA, Rifai AH, Mulsant B, Reynolds CF 3rd. Rating chronic medical illness burden in geropsychiatric practice and research: application of the cumulative illness rating scale. Psychiatry Res. 1992;41:237-48.

25. Roberts HC, Denison HJ, Martin HJ, Patel HP, Syddall H, Cooper C, Sayer AA. A review of the measurement of grip strength in clinical and epidemiological studies: towards a standardised approach. Age Ageing. 2011;40:423-9. https://doi.org/10.1093/ageing/afr051.

26. Sergi G, De Rui M, Veronese N, Bolzetta F, Berton L, Carraro S, Bano G, Coin A, Manzato E, Perissinotto E. Assessing appendicular skeletal muscle mass with bioelectrical impedance analysis in free-living Caucasian older adults. Clin Nutr. 2015;34:667-73. https://doi.org/10.1016/j.clnu.2014.07.010.

27. Guralnik JM, Simonsick EM, Ferrucci L, Glynn RJ, Berkman LF, Blazer DG, Scherr PA, Wallace RB. A short physical performance battery assessing lower extremity function: association with self-reported disability and prediction of mortality and nursing home admission. J Gerontol. 1994; 49:M85-94.

28. Schaeffner ES, Ebert N, Delanaye P, Frei U, Gaedeke J, Jakob O, Kuhlmann MK, Schuchardt M, Tölle M, Ziebig R, van der Giet M, Martus P. Two novel equations to estimate kidney function in persons aged 70 years or older. Ann Intern Med. 2012;157:471-81. https://doi.org/10.7326/0003-4819-157-7201210020-00003

29. Levey AS, Stevens LA, Schmid CH, Zhang YL, Castro AF 3rd, Feldman HI, Kusek JW, Eggers P, Van Lente F, Greene T, Coresh J. CKD-EPI (chronic kidney disease epidemiology collaboration). A new equation to estimate glomerular filtration rate. Ann Intern Med. 2009;150:604-12.

30. Pottel H, Hoste L, Dubourg L, Ebert N, Schaeffner E, Eriksen BO, Melsom T, Lamb EJ, Rule AD, Turner ST, Glassock RJ, De Souza V, Selistre L, Mariat C, Martens $F$, Delanaye P. An estimated glomerular filtration rate equation for the full age spectrum. Nephrol Dial Transplant. 2016;31:798-806. https://doi. org/10.1093/ndt/gfv454.

31. Levin A, Stevens PE, Bilous RW, Coresh J, De Francisco ALM, KE DJPEG, Hemmelgarn BR, Iseki K, Lamb EJ, Levey AS, Riella MC, Shlipak MG, Wang H, White CT, Winearls CG. Kidney disease: improving global outcomes (KDIGO) CKD work group. KDIGO 2012 clinical practice guideline for the evaluation and management of chronic kidney disease. Kidney Int Suppl. 2013;3:1-150. https://doi.org/10.1038/kisup.2012.73.

32. Curcio F, Basile C, Liguori I, Della-Morte D, Gargiulo G, Galizia G, Testa G, Langellotto $A$, Cacciatore $F$, Bonaduce $D$, Abete $P$. Tinetti mobility test is related to muscle mass and strength in non-institutionalized elderly people. Age (Dordr). 2016;38(5-6):525-33. https://doi.org/10.1007/s11357-016-9935-9 Epub 2016 Aug 26.

33. Ungar A, Mussi C, Ceccofiglio A, Bellelli G, Nicosia F, Bo M, Riccio D, Martone AM, Guadagno L, Noro G, Ghidoni G, Rafanelli M, Marchionni N, Abete P. Etiology of Syncope and unexplained falls in elderly adults with dementia: Syncope and dementia (SYD) study. J Am Geriatr Soc. 2016;64(8):1567-73. https://doi.org/10.1111/jgs.14225 Epub 2016 Jun 28.

34. Sobestiansky S, Michaelsson K, Cederholm T. Sarcopenia prevalence and associations with mortality and hospitalisation by various sarcopenia definitions in 85-89 year old community-dwelling men: a report from the ULSAM study. BMC Geriatr. 2019;19:318. https://doi.org/10.1186/s12877-0191338-1.
35. Bachettini NP, Bielemann RM, Barbosa-Silva TG, Menezes AMB, Tomasi E, Gonzalez MC. Sarcopenia as a mortality predictor in community-dwelling older adults: a comparison of the diagnostic criteria of the European Working Group on Sarcopenia in Older People. Eur I Clin Nutr. 2019. https:// doi.org/10.1038/s41430-019-0508-8, https://doi.org/10.1038/s41430-0190508-8.

36. Foley RN, Wang C, Ishani A, Collins AJ, Murray AM. Kidney function and sarcopenia in the United States general population: NHANES III. Am J Nephrol. 2007;27:279-86. https://doi.org/10.1159/000101827.

37. Sharma D, Hawkins M, Abramowitz MK. Association of sarcopenia with eGFR and misclassification of obesity in adults with CKD in the United States. Clin J Am Soc Nephrol. 2014;9:2079-88. https://doi.org/10.2215/CJN.02140214.

38. Kim JE, Lee YH, Huh JH, Kang DR, Rhee Y, Lim SK. Early-stage chronic kidney disease, insulin resistance, and osteoporosis as risk factors of sarcopenia in aged population: the fourth Korea National Health and nutrition examination survey (KNHANES IV), 2008-2009. Osteoporos Int. 2014;25:218998. https://doi.org/10.1007/s00198-014-2745-y.

39. Pereira RA, Cordeiro AC, Avesani CM, Carrero JJ, Lindholm B, Amparo FC, Amodeo C, Cuppari L, Kamimura MA. Sarcopenia in chronic kidney disease on conservative therapy: prevalence and association with mortality. Nephrol Dial Transplant. 2015;30:1718-25. https://doi.org/10.1093/ndt/gfv133.

40. Moon SJ, Kim TH, Yoon SY, Chung JH, Hwang HJ. Relationship between stage of chronic kidney disease and sarcopenia in Korean aged 40 years and older using the Korea National Health and nutrition examination surveys (KNHANES IV-2, 3, and V-1, 2), 2008-2011. PLoS One. 2015;10: e0130740. https://doi.org/10.1371/journal.pone.0130740.

41. Dierkes J, Dahl H, Lervaag Welland N, Sandnes K, Sæle K, Sekse I, Marti HP. High rates of central obesity and sarcopenia in CKD irrespective of renal replacement therapy - an observational cross-sectional study. BMC Nephrol. 2018;19:259. https://doi.org/10.1186/s12882-018-1055-6 Erratum in: BMC Nephrol. 2018 Dec 24;19(1):375. PMID: 30305034; PMCID: PMC6180401.

42. D'Alessandro C, Piccoli GB, Barsotti M, Tassi S, Giannese D, Morganti R, Cupisti A. Prevalence and correlates of sarcopenia among elderly CKD outpatients on tertiary care. Nutrients. 2018;10:1951. https://doi.org/10.3390/ nu10121951.

43. Raman M, Middleton RJ, Kalra PA, Green D. Estimating renal function in old people: an in-depth review. Int Urol Nephrol. 2017:49:1979-88. https://doi. org/10.1007/s11255-017-1682-z.

44. Han E, Lee YH, Kim G, Kim SR, Lee BW, Kang ES, Ahn CW, Cha BS. Sarcopenia is associated with albuminuria independently of hypertension and diabetes: KNHANES 2008-2011. Metabolism. 2016;65:1531-40. https:// doi.org/10.1016/j.metabol.2016.07.003.

45. Bouchi R, Fukuda T, Takeuchi T, Minami I, Yoshimoto T, Ogawa Y. Sarcopenia is associated with incident albuminuria in patients with type 2 diabetes: a retrospective observational study. J Diabetes Investig. 2017;8: 783-7. https://doi.org/10.1111/jdi.12636.

46. Lim SY, Lee KB, Kim H, Hyun YY. Low skeletal muscle mass predicts incident dipstick albuminuria in Korean adults without chronic kidney disease: a prospective cohort study. Nephron. 2019;141:105-11. https://doi.org/10. 1159/000494392.

\section{Publisher's Note}

Springer Nature remains neutral with regard to jurisdictional claims in published maps and institutional affiliations.

Ready to submit your research? Choose BMC and benefit from:

- fast, convenient online submission

- thorough peer review by experienced researchers in your field

- rapid publication on acceptance

- support for research data, including large and complex data types

- gold Open Access which fosters wider collaboration and increased citations

- maximum visibility for your research: over $100 \mathrm{M}$ website views per year

At BMC, research is always in progress.

Learn more biomedcentral.com/submission 